\title{
A spectral alternative for continuous families of self-adjoint operators
}

\author{
Alexander Y. Gordon
}

\begin{abstract}
We consider a continuous family of self-adjoint operators $A_{S}$ in a separable Hilbert space, the parameter $s$ being a point of a complete metric space $S$. It is well known that isolated simple eigenvalues (assuming that the operators are bounded and the mapping $s \mapsto A_{S}$ is continuous in the norm sense) behave "well": under small changes of the parameter they do not disappear and change continuously. Unlike this, the eigenvalues embedded in the essential spectrum can display a very "bad" behavior. It turns out, nevertheless, that if the set of eigenvalues is non-empty for a topologically rich (e.g., open) set of values of the parameter, then the (multi-valued) eigenvalue function has continuous branches.

One application is as follows. Suppose a one-dimensional quasi-periodic Schrödinger operator has Cantor spectrum; then a Baire generic operator in its hull does not have eigenvalues.
\end{abstract}

Mathematics Subject Classification (2010). Primary 47A56, 47B25; Secondary 34L40, 47E05.

Keywords. Self-adjoint operator, operator family, eigenvalue, continuous branch, Cantor spectrum, ergodic operator.

\section{Introduction}

Let $\left\{A_{s}\right\}_{s \in S}$ be a family of self-adjoint operators in a separable Hilbert space $\mathscr{H}$ parametrized by a complete metric space $S$. We need to assume some kind of continuity of the mapping $s \mapsto A_{s}$. Let first the operators $A_{s}$ be of the form

$$
A_{s}=A+B_{s}, \quad s \in S,
$$

where $A$ is a self-adjoint operator (bounded or not) and $B_{S}$ is a bounded self-adjoint operator that depends on $s$ strongly continuously. Here are two examples.

(a) Rank one perturbations:

$$
A_{s}=A+s P, \quad s \in \mathbb{R},
$$

where $A$ is a cyclic self-adjoint operator and $P=(\cdot, \varphi) \varphi$ is the orthogonal projection onto a one-dimensional cyclic subspace $\mathbb{C} \varphi(\|\varphi\|=1)$ of $A$. 
(b) Almost periodic Schrödinger operators in $L^{2}(\mathbb{R})$ or $l^{2}(\mathbb{Z})$ :

$$
A_{s}=-\Delta+v_{s}, \quad s \in \Omega,
$$

where $\Delta$ is the one-dimensional Laplacian or lattice Laplacian, $\Omega$ is the Bohr compact of an almost periodic real-valued function $v(\cdot)$, and $v_{s}(\cdot)$ is the element of its hull corresponding to $s \in \Omega$; the second summand in (3) is the operator of multiplication by the function $v_{S}(\cdot)$.

In many cases the parameter space $S$ is also endowed with a natural Borel measure, $\mu$. In (a), it is the Lebesgue measure on $\mathbb{R}$; in (b), it is the Haar measure on $\Omega$. In the theory of random and almost periodic operators (see [5] and [19]) it often turns out that for $\mu$-almost every $s \in S$ the spectrum of the operator (1) is pure point (i.e., there exists an orthonormal basis consisting of eigenvectors). In the case of rank one perturbations (2), a necessary and sufficient condition for the spectrum to be pure point for almost all $s \in \mathbb{R}$ is given in [22]. Applications of that result to random operators include the one-dimensional Anderson model (see [22]), where, under mild conditions, the spectrum turns out to be pure point for a.e. $s \in \mathbb{R}$. The almost periodic operator (3) in $l^{2}(\mathbb{Z})$, in the case of the celebrated almost Mathieu potential (see [16])

$$
v_{s}(n)=\lambda \cos (2 \pi \alpha n+s), \quad s \in \mathbb{R} / 2 \pi \mathbb{Z},
$$

has pure point spectrum for almost all $s$ if the irrational $\alpha$ is Diophantine and $|\lambda|>2$, see [14]. In both these examples the eigenvalues embedded in the essential spectrum (which has the cardinality of the continuum) are dense there. The same holds in a number of other cases, see [3] and references therein.

Therefore, if by "small" sets we understand those subsets of $S$ whose $\mu$-measure is zero, and remove a suitable one, then for the remaining "measure typical" $s$ in these examples the operator $A_{s}$ has a pure point spectrum and the eigenvalues embedded in the essential spectrum are dense in it.

On the other hand, since $S$ is a complete metric space, there is another notion of "smallness" for its subsets: we can consider as small the first category sets (also called meager sets), i.e., countable unions of nowhere dense sets. What structure of the spectrum (if any) is Baire generic, i.e., holds for all $s$ except those in some meager set (or equivalently, for all $s$ in a dense $G_{\delta}$ set)?

In the cases, where the answer is known, it is quite different from the above one. In particular, in examples (2) and (3), (4) for a Baire generic $s$ there are no eigenvalues embedded in the essential spectrum. (For the operator family (2), this is proved in [12] and independently in [7]; for the family (3), (4), this follows from the result of [15].)

Note that these results on absence of eigenvalues in the essential spectrum for rank one perturbations (2) and for almost periodic operators (3), (4) are of the same kind. Nevertheless, the proofs in these two cases are completely different and do not reveal any common mechanism behind these phenomena.

In the present work we find an obstruction that in many cases prevents the existence of eigenvalues embedded in the essential spectrum for all values of the parameter 
(even if they are dense there for almost all values of the parameter with respect to a suitable measure). Now the results of [12] and [7], on the one hand, and of [15], on the other, get a common explanation.

The families of self-adjoint operators that we consider in this work do not necessarily have the form (1). The only requirement is that $A_{s}$ depend on $s$ continuously in the strong resolvent sense. In the rest of the paper, the term "Baire generic" is abbreviated to "generic". A property of an element of a complete metric space $S$ is, therefore, generic if there exists a dense $G_{\delta}$ subset of $S$ all of whose elements have that property.

The remaining part of the paper is organized as follows. In Section 2 we state the main result - Theorem 1. It is proved in Section 3, which also contains some generalizations of the main result. Section 4 provides additional information (Theorem 2) about eigenvectors in the case where the eigenvalues are simple. Section 5 contains a sufficient condition for genericity of purely continuous spectrum (Theorem 3). In Section 6 some applications are considered. First we prove the genericity of absence of eigenvalues for certain ergodic families of one-dimensional Schrödinger operators with Cantor spectrum. Then we show how results obtained in [12], [7] and in [15] can be derived from the results of this work.

Acknowledgements. I am indebted to Artur Avila for his simplification of the original proof of Theorem 1. I am also grateful to Yoram Last and Barry Simon for valuable discussions and to Svetlana Jitomirskaya for useful suggestions regarding applications of the main result. Finally, it is a pleasure to acknowledge the influence of the work of Ilya Goldsheid [9] (see also [10]) on this paper.

\section{The main result}

Theorem 1. Let $S$ be a complete metric space and $\left\{A_{S}\right\}_{s \in S}$ be a family of self-adjoint operators in a separable Hilbert space $\mathscr{H}$ such that the mapping $s \mapsto A_{s}$ is continuous in the strong resolvent sense (that is, the mapping $S \ni s \mapsto\left(A_{s}-z_{0} I\right)^{-1} x \in \mathscr{H}$ is continuous for any $z_{0} \in \mathbb{C} \backslash \mathbb{R}$ and any $\left.x \in \mathcal{H}\right)$. Let $F$ be a closed subset of the real line $\mathbb{R}$. Denote by $Z$ the set of all $s \in S$ such that the operator $A_{s}$ has at least one eigenvalue in $F$.

An alternative takes place: either

(i) $Z$ is a meager set, or

(ii) $Z$ has a non-empty interior; in this case there exist a non-empty open set $U \subset Z$ and a continuous function

$$
s \longmapsto \mathcal{E}_{S}: U \longrightarrow F
$$

such that, for all $s \in U, \mathcal{E}_{s}$ is an eigenvalue of $A_{s}$. 
Remarks. 1. The first part of this theorem (stating that either $Z$ is meager, or it contains an open set) can also be derived from [21] (Theorem 1.1). What we emphasize is the existence, in the latter case, of an eigenvalue that depends continuously on the parameter (on some smaller open set).

2. Let the operator $A_{s}$ be bounded for all $s$ and depend on $s$ continuously in the norm sense. Assume that, for some $s=s_{0}, A_{s}$ has a simple isolated eigenvalue $E_{0}$. Then, as is well known, for all $s$ close enough to $s_{0}, A_{s}$ also has a simple isolated eigenvalue $E(s)$ close to $E_{0}$; it is continuous in $s$ and satisfies $E\left(s_{0}\right)=E_{0}$. (The corresponding eigenspace may be determined as the range of a suitable Riesz projection.) So in this case our statement is obvious. But if the eigenvalues are not isolated - e.g., if they are dense in some interval, - we do not have such a natural way to identify individual eigenvalues for different values of $s$, and it is not clear whether it is possible to pick a point $E(s)$ in this dense set (depending on $s$ ) that will be continuous in $s$ on some open subset of $S$. Of course, such a selection is impossible if for all $s$ in some dense subset of $S$ the operator $A_{s}$ has no eigenvalues at all. The theorem implies that this is the only case where continuous selection of an eigenvalue is impossible.

\section{The proof}

1. Consider the topological product

$$
\mathcal{T}:=S \times F \times \mathscr{H},
$$

where the Hilbert space $\mathscr{H}$ is endowed with the weak topology. Furthermore, for any $m \in \mathbb{N}$ define a topological subspace of $\mathcal{T}$

$$
\mathcal{T}_{m}:=S \times F_{m} \times B_{1},
$$

where $F_{m}=F \cap[-m, m]$ and $B_{1}=\{y \in \mathscr{H} \mid\|y\| \leq 1\}$. Note that $\mathcal{T}_{m}$ endowed with the induced topology is a product of two completely metrizable spaces $S$ and $F_{m} \times B_{1}$, the latter being compact.

Also consider a subset of $\mathcal{T}$

$$
\text { Sol }:=\left\{\eta=(s, E, y) \in \mathcal{T} \mid A_{s} y=E y, y \neq 0\right\} .
$$

Clearly,

$$
\operatorname{pr}_{S}(\mathrm{Sol})=Z
$$

(by $\operatorname{pr}_{S}, \operatorname{pr}_{S \times F}$, etc. we denote the corresponding projections of the product $\mathcal{T}=$ $S \times F \times \mathscr{H}$ onto $S, S \times F$, etc.). Furthermore, fix a sequence $\left\{g_{k}\right\}_{k=1}^{\infty}$ that is dense in the unit sphere of $\mathscr{H}$; let

$$
\mathrm{Sol}_{m}^{k}:=\left\{\eta=(s, E, y) \in \mathcal{T} \mid A_{s} y=E y,\left(y, g_{k}\right)=\frac{3}{4}, y \in B_{1}, E \in F_{m}\right\}
$$


or, equivalently,

$$
\operatorname{Sol}_{m}^{k}:=\operatorname{Sol} \cap\left\{\eta=(s, E, y) \in \mathcal{T}_{m} \mid\left(y, g_{k}\right)=\frac{3}{4}\right\} .
$$

2. We will need the following simple fact.

Lemma 1. Sol $_{m}^{k}$ is closed in $\mathcal{T}_{m}$.

Proof. Suppose

$$
\operatorname{Sol}_{m}^{k} \ni \eta_{j}=\left(s_{j}, E_{j}, y_{j}\right) \longrightarrow \eta=(s, E, y) \in \mathcal{T}_{m} \quad \text { as } j \rightarrow \infty .
$$

We should verify that $\eta \in \operatorname{Sol}_{m}^{k}$. That $\left(y, g_{k}\right)=\frac{3}{4}$ is obvious, since $y_{j} \stackrel{w}{\rightarrow} y$ as $j \rightarrow \infty$ (by $\stackrel{w}{\rightarrow}$ we denote the weak convergence in $\mathscr{H}$ ).

According to (6), it remains to check that $\eta \in$ Sol, i.e., $A_{s} y=E y$, or equivalently, $\left(A_{s}-z_{0} I\right)^{-1} y=\left(E-z_{0}\right)^{-1} y$, where $z_{0} \in \mathbb{C} \backslash \mathbb{R}$ is fixed. We have $A_{s_{j}} y_{j}=E_{j} y_{j}$, hence

$$
\left(A_{s_{j}}-z_{0} I\right)^{-1} y_{j}=\left(E_{j}-z_{0}\right)^{-1} y_{j} .
$$

The left-hand side converges weakly to $\left(A_{s}-z_{0} I\right)^{-1} y$. (Indeed, if $\varphi$ is an arbitrary vector in $\mathscr{H}$, then $\left(\left(A_{s_{j}}-z_{0} I\right)^{-1} y_{j}, \varphi\right)=\left(y_{j},\left(A_{s_{j}}-\overline{z_{0}} I\right)^{-1} \varphi\right)$ converges to $\left(y,\left(A_{s}-\overline{z_{0}} I\right)^{-1} \varphi\right)$, in view of the strong continuity of the resolvent of $A_{s}$ in $s$.) At the same time, the right-hand side of (7) converges weakly to $\left(E-z_{0}\right)^{-1} y$. Thus $\left(A_{s}-z_{0} I\right)^{-1} y=\left(E-z_{0}\right)^{-1} y$.

3. Let

$$
Z_{m}^{k}:=\operatorname{pr}_{S}\left(\operatorname{Sol}_{m}^{k}\right)
$$

The set $Z_{m}^{k}$ is closed in $S$.

This is a special case of the following general statement.

Lemma 2. If $M$ and $N$ are topological spaces, $N$ is compact and $B$ is a closed subset of $M \times N$, then the projection of $B$ to $M$ is closed.

Assume that case (i) of the theorem does not take place, i.e., the set $Z$ is not a countable union of nowhere dense sets. Since

$$
Z=\bigcup_{m, k=1}^{\infty} Z_{m}^{k}
$$

there is a pair $(k, m) \in \mathbb{N}^{2}$ such that $Z_{m}^{k}$ is not nowhere dense in $S$. Being closed, $Z_{m}^{k}$ contains some open set $U$; this implies the first part of (ii). 
4. Now we are going to derive the existence of an eigenvalue $\mathcal{E}_{s} \in F$ of $A_{s}$ depending continuously on $s \in Z_{m}^{k}$.

Lemma 3. If $\eta=(s, E, y) \in \operatorname{Sol}_{m}^{k}$ and $\tilde{\eta}=(s, \widetilde{E}, \tilde{y}) \in \operatorname{Sol}_{m}^{k}$, then $E=\widetilde{E}$.

Proof. We have $A_{s} y=E y$ and $A_{s} \tilde{y}=\widetilde{E} \tilde{y}$, so that if $E \neq \widetilde{E}$, then $y$ and $\tilde{y}$ are orthogonal. It follows then from the Bessel inequality and relations $\left(y, g_{k}\right)=$ $\left(\tilde{y}, g_{k}\right)=\frac{3}{4},\|y\| \leq 1$, and $\|\tilde{y}\| \leq 1$ that

$$
\left\|g_{k}\right\|^{2} \geq\left|\left(g_{k}, \frac{y}{\|y\|}\right)\right|^{2}+\left|\left(g_{k}, \frac{\tilde{y}}{\|\tilde{y}\|}\right)\right|^{2} \geq\left(\frac{3}{4}\right)^{2}+\left(\frac{3}{4}\right)^{2}>1,
$$

which is impossible because $\left\|g_{k}\right\|=1$.

Lemma 3 implies that on the set

$$
G_{m}^{k}:=\operatorname{pr}_{S \times F_{m}}\left(\operatorname{Sol}_{m}^{k}\right)
$$

the second component of a pair $(s, E)$ is uniquely determined by its first component. In other words, $G_{m}^{k}$ is the graph of a function

$$
s \longmapsto \mathcal{E}_{s}: Z_{m}^{k} \longrightarrow F_{m}
$$

(we use the fact that $\operatorname{pr}_{S}\left(G_{m}^{k}\right)=\operatorname{pr}_{S}\left(\operatorname{Sol}_{m}^{k}\right)=Z_{m}^{k}$ ).

The set $G_{m}^{k}$ is closed, again by Lemma 2, so that the function (9) has a closed graph; consequently, it is continuous, in view of the following lemma.

Lemma 4. If $M$ and $N$ are topological spaces, $N$ is compact and a mapping $f: M \rightarrow N$ has a closed graph, then $f$ is continuous.

As was shown, if case (i) of Theorem 1 does not take place, then the set $Z_{m}^{k}$ (for some $m$ and $k$ ) contains a non-empty open set $U$. The restriction of the continuous function (9) to $U$ is continuous as well. This completes the proof of Theorem $1 .{ }^{1}$

Remarks. 1 . The closed set $F$ in the formulation of Theorem 1 can be replaced by an arbitrary $F_{\sigma}$ set, in particular, by any open set. This is an immediate consequence of the theorem.

2. The set $F$ in Theorem 1 does not need to be fixed. The following generalization of Theorem 1 is true.

Theorem 1*. Let $\left\{A_{s}\right\}_{s \in S}$ be a family of self-adjoint operators satisfying the conditions of Theorem 1. Let $\mathcal{F}$ be a closed subset of the product $S \times \mathbb{R}$; denote by $\mathcal{F}_{s}(s \in S)$ its (closed) cross-section

$$
\mathscr{F}_{s}:=\{E \in \mathbb{R} \mid(s, E) \in \mathscr{F}\}
$$

\footnotetext{
${ }^{1}$ The proof presented here is a simplified version of the original proof; the simplification is due to Artur Avila.
} 
and by $Z$ the set of all $s \in S$ for which the operator $A_{s}$ has at least one eigenvalue in $\mathcal{F}_{s}$. Then either

(i) $Z$ is a meager set, or

(ii) there exist a non-empty open set $U \subset Z$ and a continuous function

$$
s \longmapsto \mathcal{E}_{S}: U \longrightarrow \mathbb{R}
$$

such that for all $s \in U$ we have: $\mathcal{E}_{S}$ is an eigenvalue of $A_{s}$ and $\varepsilon_{s} \in \mathcal{F}_{S}$.

The proof differs from that of Theorem 1 only in that now we define the topological space $\mathcal{T}$ by $\mathcal{T}:=\mathcal{F} \times \mathscr{H}$ and then define $\mathcal{T}_{m}$ as follows: $\mathcal{T}_{m}:=\mathcal{F}_{m} \times B_{1}$, where $\widetilde{F}_{m}=\{(s, E) \in \mathscr{F}|| E \mid \leq m\}$.

Corollary 1. Let $A_{s}=A+B_{s}$, where $A$ is a self-adjoint operator in a separable Hilbert space $\mathcal{H}$ and $B_{s}$ is a bounded self-adjoint operator in $\mathscr{H}$ depending on the point $s$ of a complete metric space $S$ continuously in the norm sense. Denote by $\sigma_{\mathrm{ess}}\left(A_{s}\right)$ the essential spectrum of $A_{s}$ (which in general depends on $s$ ). Then either

(i) for a generic $s \in S$ there are no eigenvalues of $A_{S}$ in $\sigma_{\mathrm{ess}}\left(A_{S}\right)$, or

(ii) there is a non-empty open set $U \subset S$ and an eigenvalue $\varepsilon_{s}$ of $A_{s}(s \in U)$ that belongs to $\sigma_{\mathrm{ess}}\left(A_{S}\right)$ and depends continuously on $s \in U$.

Proof. In order to apply Theorem $1^{*}$, we need to verify that the set $\mathcal{F}:=\{(s, E) \in$ $\left.S \times \mathbb{R} \mid E \in \sigma_{\text {ess }}\left(A_{s}\right)\right\}$ is closed. This follows immediately from the following characterization of the essential spectrum [8]: $E \in \sigma_{\mathrm{ess}}\left(A_{S}\right)$ if and only if for any $\varepsilon>0$ there exists an infinitely-dimensional linear set $D \subset D\left(A_{s}\right)$ such that $\left\|A_{s} x-E x\right\| \leq \varepsilon\|x\|$ for all $x \in D$.

\section{The case of simple eigenvalues}

Theorem 2. Let $\left\{A_{s}\right\}_{s \in S}$ be a family of operators that satisfies the conditions of Theorem 1, and $F$ a closed subset of $\mathbb{R}$. Assume, in addition, that all eigenvalues of the operators $A_{s}$ are simple. Then an alternative takes place: either

(i) for all $s$ in some dense $G_{\delta}$ set, the operator $A_{s}$ has no eigenvalues in $F$, or

(ii) there exist a non-empty open set $U \subset Z$ and two mappings $U \ni s \mapsto \mathcal{E}_{s} \in F$ and $U \ni s \mapsto \mathcal{Y}_{s} \in \mathcal{H} \backslash\{0\}$ such that

(a) $A_{s} y_{s}=\varepsilon_{s} y_{s}$ for all $s \in U$;

(b) the mapping $s \mapsto \mathcal{E}_{S}$ is continuous;

(c) the mapping $s \mapsto \mathcal{Y}_{s}$ is weakly continuous;

(d) there is a dense $G_{\delta}$ set $Q$ in $U$ such that for any $s^{*} \in Q$ we have $\left\|y_{s}-y_{s^{*}}\right\| \rightarrow 0$ as $U \ni s \rightarrow s^{*}$. 
Proof. Assume that case (ii) of Theorem 1 takes place. In what follows, we use the objects and notation introduced in the proof of that theorem. For each point $s$ of $Z_{m}^{k}=\operatorname{pr}_{S}\left(\operatorname{Sol}_{m}^{k}\right)$, there is only one triple $(s, E, y) \in \operatorname{Sol}_{m}^{k}$ with the given $s$. (The uniqueness of $E=\mathcal{E}_{s}$ was established in the proof of Theorem 1; the uniqueness of $y$, denoted below by $y_{s}$, follows from the equality $\left(y, g_{k}\right)=\frac{3}{4}$ (see (5)) and the assumed simplicity of the eigenvalue $\mathcal{E}_{s}$ of $A_{s}$.)

Consequently, the set $\mathrm{Sol}_{m}^{k}$ is the graph of a mapping $Z_{m}^{k} \ni s \mapsto\left(\mathcal{E}_{s}, y_{s}\right) \in$ $F_{m} \times B_{1}$, which is continuous by Lemmas 1 and 4 . In particular, the mapping $Z_{m}^{k} \ni$ $s \mapsto y_{s} \in B_{1}$ is weakly continuous, so that its restriction to the open subset $U$ of $Z_{m}^{k}$ is weakly continuous as well. Therefore, the mapping $U \ni s \mapsto\left(\mathcal{E}_{s}, y_{s}\right) \in F_{m} \times B_{1}$ has properties (a), (b) and (c). Obviously, $y_{s} \neq 0$. It remains to prove (d).

Fix an orthonormal basis $e_{1}, e_{2}, \ldots$ of the Hilbert space $\mathscr{H}$. The function $h(s):=$ $\left\|y_{s}\right\|^{2}, s \in Z_{m}^{k}$, is the pointwise limit of a sequence of continuous functions: $h(s)=$ $\lim _{n \rightarrow \infty} h_{n}(s)$, where

$$
h_{n}(s)=\sum_{j=1}^{n}\left|\left(y_{s}, e_{j}\right)\right|^{2} .
$$

Consequently, there is a dense $G_{\delta}$ set in $Z_{m}^{k}$, say $X$, such that the function $h(\cdot)$ is continuous at all points of $X$ ([18], Theorem 7.3).

If $s^{*} \in X$, then, as $Z_{m}^{k} \ni s \rightarrow s^{*}$, we have $y_{s} \stackrel{w}{\rightarrow} y_{s^{*}}$ and $\left\|y_{s}\right\| \rightarrow\left\|y_{s^{*}}\right\|$, which implies $\left\|y_{s}-y_{s^{*}}\right\| \rightarrow 0$. Setting $Q:=X \cap U$ completes the proof.

\section{A sufficient condition for genericity of purely continuous spectrum}

Theorem 3. Let $\left\{A_{s}\right\}_{s \in S}$ be an operator family satisfying the conditions of Theorem 1 . Let $F$ be a closed subset of $\mathbb{R}$. Denote by $D$ the set of all eigenvalues of all the operators $A_{s}: D:=\cup_{s \in S} \sigma_{\mathrm{p}}\left(A_{s}\right)$. Suppose that

(a) $S$ is locally connected;

(b) the subset $F \cap D$ of $\mathbb{R}$ has no interior points;

(c) for any $E \in F$, the set

$$
N_{E}:=\left\{s \in S: E \text { is an eigenvalue of } A_{s}\right\}
$$

has no interior points.

Then for all s in some dense $G_{\delta}$ set $S_{0} \subset S$, the operator $A_{S}$ has no eigenvalues in $F$.

Proof. Assume the converse. Then case (ii) of Theorem 1 takes place, so that there exist an open set $U \subset S$ and a continuous function $s \mapsto \mathcal{E}_{s}: U \rightarrow F$ such that $\mathcal{E}_{s}$ is an eigenvalue of $A_{s}$ for all $s \in U$. Let $U_{0}$ be a connected component of $U$. By (a), it is open. Thus, we have a continuous function $U_{0} \ni s \mapsto \mathcal{E}_{s} \in D \cap F$ and, since $U_{0}$ 
is connected, the set $\left\{\varepsilon_{s}: s \in U_{0}\right\} \subset D \cap F$ is connected as well, being, therefore, an interval. By the assumption (b), this interval consists of a single point, say $E_{0}$. This implies that the set (10) with $E=E_{0}$ contains an open set, $U_{0}$. However, this contradicts (c).

\section{Applications}

\subsection{Ergodic one-dimensional Schrödinger operators with Cantor spectrum}

6.1.1. Continuum operators. Let $S$ be a complete metric space; $\left\{G^{t}\right\}_{t \in \mathbb{R}}$ a group of its homeomorphisms such that $G^{t}(s)$ is continuous in $t$ for any $s ; V(\cdot)$ a bounded continuous real-valued function on $S$; and $\mu$ a Borel measure on $S$ such that $\mu(S)=1$. For $s \in S$, define a self-adjoint operator $H_{S}$ on $L^{2}(\mathbb{R})$ by

$$
H_{s}=-\frac{d^{2}}{d t^{2}}+v_{s},
$$

where $v_{s}$ is the operator of multiplication by the function

$$
v_{s}(t)=V\left(G^{t}(s)\right) \text {. }
$$

Suppose that

(A) the group $\left\{G^{t}\right\}_{t \in \mathbb{R}}$ preserves the measure $\mu$ and is $\mu$-ergodic;

(B) the group is minimal, that is, the trajectory $\left\{G^{t}(s)\right\}_{t \in \mathbb{R}}$ of any point $s \in S$ is dense in $S$;

(C) $\mu(U)>0$ for any non-empty open set $U \subset S$.

The operators $H_{s}$ and $H_{G^{\tau}(s)}$ are unitarily equivalent: $H_{G^{\tau}(s)}=U_{\tau}^{-1} H_{s} U_{\tau}$, where $\left(U_{\tau} y\right)(t)=y(t-\tau)$; hence their spectra $\sigma\left(H_{s}\right)$ and $\sigma\left(H_{G^{\tau}(s)}\right)$ are identical. Furthermore, the multiplication operator $v_{s}$ depends on $s$ strongly continuously, so that the operator family (11) satisfies the strong resolvent continuity condition: if $s_{k} \rightarrow s$, then $H_{s_{k}}$ converges to $H_{s}$ in the strong resolvent sense ([20], Theorem VIII.25). Consequently, for any $E \in \sigma\left(H_{s}\right)$ there exist $E_{k} \in \sigma\left(H_{s_{k}}\right)$ such that $E_{k} \rightarrow E$ ([20], Theorem VIII.24). Assumption (B), therefore, implies that the spectrum of $H_{s}$ does not depend on $s$. Denote this closed set by $\Sigma$. It does not contain isolated points ([19], Theorem 2.11).

Theorem 4. Suppose that the group $\left\{G^{t}\right\}_{t \in \mathbb{R}}$, the measure $\mu$ and the function $V$ satisfy the above assumptions. If $S$ is locally connected and $\Sigma$ is nowhere dense in $\mathbb{R}$, then there is a dense $G_{\delta}$ set $S_{0} \in S$ such that for any $s \in S_{0}$ the operator $H_{S}$ defined by (11) and (12) has no eigenvalues. 
Proof. We use Theorem 3, putting $F:=\Sigma$. As was said, the operator family (11) satisfies the strong resolvent continuity condition. Conditions (a) and (b) of Theorem 3 are fulfilled due to the assumptions of Theorem 4. Condition (c) is satisfied, because the operators (11) cannot have eigenvalues of infinite multiplicity, so that the set (10) has $\mu$-measure zero ([19], Theorem 2.12) and, in view of (C), cannot contain an open set.

Corollary 2. Let $H_{s}$, where $s=\left(s_{1}, \ldots, s_{d}\right) \in \mathbb{T}^{d}=\mathbb{R}^{d} / \mathbb{Z}^{d}(d \geq 2)$, be the operator $(11)$ in $L^{2}(\mathbb{R})$ with the potential

$$
v_{s}(t)=V\left(s_{1}+\alpha_{1} t, \ldots, s_{d}+\alpha_{d} t\right), \quad t \in \mathbb{R} ;
$$

here $V$ is a continuous real-valued function on $\mathbb{T}^{d}$, and the numbers $\alpha_{1}, \ldots, \alpha_{d}$ are rationally independent. If the spectrum of the operator $H_{s}$ is nowhere dense (for some $s$ and hence for all $s \in \mathbb{T}^{d}$ ), then for all $s$ in some dense $G_{\delta}$ set $S_{0} \subset \mathbb{T}^{d}$ the operator $H_{S}$ has no eigenvalues.

Proof. Apply Theorem 4, setting $S=\mathbb{T}^{d}$ and defining the homeomorphism

$$
G^{t}: \mathbb{T}^{d} \longrightarrow \mathbb{T}^{d} \quad(t \in \mathbb{R})
$$

by $G^{t}\left(s_{1}, \ldots, s_{d}\right)=\left(s_{1}+\alpha_{1} t, \ldots, s_{d}+\alpha_{d} t\right), \mu$ being the Lebesgue measure on $\mathbb{T}^{d}$.

\subsubsection{Discrete operators}

1. Let $S$ be a complete metric space, $G$ its homeomorphism, $V(\cdot)$ a bounded continuous real-valued function on $S$, and $\mu$ a Borel measure on $S$ such that $\mu(S)=1$. For $s \in S$, define a self-adjoint operator $H_{s}$ on $l^{2}(\mathbb{Z})$ by

$$
\left(H_{s} y\right)(n)=y(n-1)+y(n+1)+v_{s}(n) y(n), \quad n \in \mathbb{Z},
$$

where

$$
v_{s}(n)=V\left(G^{n}(s)\right)
$$

Suppose that

(A) the homeomorphism $G$ preserves the measure $\mu$ and is $\mu$-ergodic;

(B) $G$ is minimal, that is, the trajectory $\left\{G^{n}(s)\right\}_{n \in \mathbb{Z}}$ of any point $s \in S$ is dense in $S$;

(C) $\mu(U)>0$ for any non-empty open set $U \subset S$.

All the operators $H_{S}(s \in S)$ have the same spectrum, from now on denoted by $\Sigma$ (with interior $\operatorname{Int}(\Sigma)$ ). Furthermore, this closed set does not have isolated points. These statements, as well as the following theorem, can be proved in the same way as in the continuum case. 
Theorem 5. Suppose that the homeomorphism $G$, the measure $\mu$ and the function $V$ satisfy the above assumptions. If $S$ is locally connected and $\Sigma$ is nowhere dense in $\mathbb{R}$, then there is a dense $G_{\delta}$ set $S_{0} \in S$ such that for all $s \in S_{0}$ the operator $H_{s}$ defined by (13) and (14) has no eigenvalues.

Corollary 3. Let $H_{s}$, where $s=\left(s_{1}, \ldots, s_{d}\right) \in \mathbb{T}^{d}=\mathbb{R}^{d} / \mathbb{Z}^{d}(d \geq 1)$, be the operator (13), (14) in $l^{2}(\mathbb{Z})$, where $G$ is a shift on $\mathbb{T}^{d}$ :

$$
G\left(s_{1}, \ldots, s_{d}\right)=\left(s_{1}+\alpha_{1}, \ldots, s_{d}+\alpha_{d}\right)
$$

the numbers $1, \alpha_{1}, \ldots, \alpha_{d}$ being rationally independent. If $\operatorname{Int}(\Sigma)=\emptyset$, then for all $s$ in some dense $G_{\delta}$ set $S_{0} \subset \mathbb{T}^{d}$ the operator $H_{s}$ has no eigenvalues.

Corollary 4. Let $H_{s}$, where $s=\left(s_{1}, s_{2}\right) \in \mathbb{T}^{2}$, be the operator (13), (14) in $l^{2}(\mathbb{Z})$, where $G$ is a skew-shift on $\mathbb{T}^{2}$ :

$$
G\left(s_{1}, s_{2}\right)=\left(s_{1}+\alpha, s_{2}+s_{1}\right)
$$

with an irrational $\alpha$. If $\operatorname{Int}(\Sigma)=\emptyset$, then for all $s$ in a dense $G_{\delta}$ set $S_{0} \subset \mathbb{T}^{2}$ the operator $H_{S}$ has no eigenvalues.

2. Some ergodic Schrödinger operators having Cantor spectrum for generic values of the functional parameter are studied in [1]. These are operators over a strictly ergodic homeomorphism that fibers over an almost periodic dynamical system.

Proposition 1 ([1]). Let $S$ be a compact metric space and $G: S \rightarrow S$ a strictly ergodic homeomorphism (that is, $G$ is minimal and uniquely ergodic). Suppose that there exist an infinite compact Abelian group $K$ and an onto continuous mapping $p: S \rightarrow K$ such that $p(G(s))=p(s)+\alpha$ for some $\alpha \in K$ and all $s \in S$. Then for a generic $V \in C(S, \mathbb{R})$ the operator $H_{s}^{V}$ in $l^{2}(\mathbb{Z})$ defined by

$$
\left(H_{s}^{V} y\right)(n)=y(n-1)+y(n+1)+V\left(G^{n}(s)\right) y(n), \quad n \in \mathbb{Z},
$$

has Cantor spectrum.

Proposition 1 and Theorem 5 imply the following statement.

Corollary 5. Let $S, G, K$, and $p$ be such as in Proposition 1. Assume, in addition, that $S$ is locally connected. Then for a generic $V \in C(S, \mathbb{R})$ there exists a dense $G_{\delta}$ set $S_{V} \subset S$ such that for all $s \in S_{V}$ the operator (17) does not have eigenvalues.

Examples of particular interest include those where $G$ is a shift or a skew-shift on the torus (see the above Corollaries 3 and 4). 
Corollary 6. Let $H_{s}^{V}$, where $V \in C\left(\mathbb{T}^{d}, \mathbb{R}\right)$ and $s \in \mathbb{T}^{d}(d \geq 1)$, be the operator in $l^{2}(\mathbb{Z})$ given by

$$
\left(H_{s}^{V} y\right)(n)=y(n-1)+y(n+1)+V\left(s_{1}+n \alpha_{1}, \ldots, s_{d}+n \alpha_{d}\right) y(n), \quad n \in \mathbb{Z},
$$

the numbers $1, \alpha_{1}, \ldots, \alpha_{d}$ being rationally independent. Then for a generic $V \in$ $C\left(\mathbb{T}^{d}, \mathbb{R}\right)$ and all $s$ in some dense $G_{\delta}$ set $S_{V} \subset \mathbb{T}^{d}$ (which may depend on $V$ ) the operator $H_{s}^{V}$ has no eigenvalues.

Remark. For a different way to obtain this result, see [13]. ${ }^{2}$

Corollary 7. Let $H_{s}^{V}$, where $V \in C\left(\mathbb{T}^{2}, \mathbb{R}\right)$ and $s=\left(s_{1}, s_{2}\right) \in \mathbb{T}^{2}$, be the following operator in $l^{2}(\mathbb{Z})$ :

$$
\left(H_{s}^{V} y\right)(n)=y(n-1)+y(n+1)+V\left(G^{n}(s)\right) y(n), \quad n \in \mathbb{Z},
$$

where $G\left(s_{1}, s_{2}\right)=\left(s_{1}+\alpha, s_{2}+s_{1}\right)$ with an irrational $\alpha$. Then for a generic $V \in C\left(\mathbb{T}^{2}, \mathbb{R}\right)$ and all $s$ in some dense $G_{\delta}$ set $S_{V} \subset \mathbb{T}^{2}$ (which may depend on $V$ ) the operator $H_{s}^{V}$ has no eigenvalues.

3. Recently Goldstein and Schlag [11] proved that the spectrum is a Cantor set for quasi-periodic operators with one-frequency analytic potentials for almost all values of the frequency in the regime of positive Lyapunov exponent. Their main result (Theorem 1.1) implies the following statement.

Proposition 2 (Corollary to Theorem 1.1 in [11]). Suppose the operator family $A_{\omega}^{\alpha}(\omega \in \mathbb{T}=\mathbb{R} / \mathbb{Z})$ is given by the equation

$$
\left(A_{\omega}^{\alpha} y\right)(j)=y(j-1)+y(j+1)+V(j \alpha+\omega) y(j), \quad j \in \mathbb{Z},
$$

where $\alpha \in \mathbb{R}$ and $V(\cdot)$ is a 1-periodic real-analytic function on $\mathbb{R}$. Suppose $\alpha_{0} \in \mathbb{R}$ satisfies the Diophantine condition

$$
\left\|k \alpha_{0}\right\| \geq \frac{c}{k(1+\log k)^{a}}, \quad k=1,2, \ldots,
$$

with some $a>1, c>0$. Here $\|\cdot\|$ denotes the distance from the nearest integer. Suppose, furthermore, that the Lyapunov exponent $L\left(E, \alpha_{0}\right)$ of the operator $A_{\omega}^{\alpha_{0}}$ satisfies the inequality

$$
L\left(E, \alpha_{0}\right) \geq \gamma_{0}>0, \quad E^{\prime} \leq E \leq E^{\prime \prime} .
$$

\footnotetext{
${ }^{2}$ Note that the proof of one of lemmas in [13] (Lemma 2) is incomplete: it contains a statement about sequences of Schrödinger operators with convergent potentials and weakly convergent eigenfunctions that is not actually proved. To correct that, the end of the proof of the lemma should be replaced by an argument similar to that used in the proof of Lemma 1 above.
} 
Then there exists $\delta>0$ such that for almost all $\alpha \in\left(\alpha_{0}-\delta, \alpha_{0}+\delta\right)$ the spectrum of $A_{\omega}^{\alpha}$ (which, for irrational $\alpha$, does not depend on $\omega$ ) is nowhere dense in $\left[E^{\prime}, E^{\prime \prime}\right]$.

Proposition 2 and Corollary 3 lead to the following statement.

Theorem 6. Suppose the operator (19), where V(.) is a 1-periodic real-analytic function on $\mathbb{R}$, has strictly positive Lyapunov exponent $L\left(E, \alpha_{0}\right)>0$ for some $\alpha_{0}$ satisfying the Diophantine condition (20) and all $E$ in a compact interval $\Delta=\left[E^{\prime}, E^{\prime \prime}\right]$.

Then for almost all $\alpha$ in a small enough neighborhood of $\alpha_{0}$, the operator $A_{\omega}^{\alpha}$ has singular continuous spectrum in $\Delta$ for a dense $G_{\delta}$ set of $\omega \in \mathbb{T}$.

Proof. According to the result of Bourgain-Jitomirskaya [4], the real-analyticity of $V$ and the irrationality of $\alpha_{0}$ imply that the Lyapunov exponent $L(E, \alpha)$ is jointly continuous in $E$ and $\alpha$ at all points $\left(E, \alpha_{0}\right), E \in \mathbb{R}$. (Note that $L(E, \alpha)$, for rational $\alpha$, is understood here as the Lyapunov exponent averaged over $\omega \in \mathbb{T}$.) It follows that (21) holds for some small $\gamma_{0}>0$; moreover, for all $\alpha \in\left(\alpha_{0}-\beta, \alpha_{0}+\beta\right)$ with small enough $\beta>0$ we have

$$
L(E, \alpha) \geq \gamma_{0} / 2>0, \quad E^{\prime} \leq E \leq E^{\prime \prime} .
$$

By Proposition 2, the operator $A_{\omega}^{\alpha}$ has Cantor spectrum for almost all $\alpha \in\left(\alpha_{0}-\delta\right.$, $\left.\alpha_{0}+\delta\right)$, where $\delta \in(0, \beta]$ is sufficiently small. By Corollary 3 , for any such $\alpha$ and all $\omega$ in a dense $G_{\delta}$ set $T_{\alpha} \in \mathbb{T}$, the operator $A_{\omega}^{\alpha}$ does not have eigenvalues in $\Delta$.

The singularity of the spectrum of $A_{\omega}^{\alpha}$ in the interval $\Delta$ is implied by inequality (22); this follows from the Ishii-Pastur-Kotani theorem (see, e.g., [6]) for almost all $\omega$ and from the result of Last and Simon [17] for all $\omega$.

Corollary 8. Suppose the operator (19) with a 1-periodic real-analytic function $V$ has positive Lyapunov exponent $L(E, \alpha)>0$ for almost all $(\alpha, E) \in\left[\alpha^{\prime}, \alpha^{\prime \prime}\right] \times$ $\left[E^{\prime}, E^{\prime \prime}\right]$. Then for almost every $\alpha \in\left[\alpha^{\prime}, \alpha^{\prime \prime}\right]$ the operator $A_{\omega}^{\alpha}$ has singular continuous spectrum in $\left[E^{\prime}, E^{\prime \prime}\right]$ for a dense $G_{\delta}$ set of $\omega \in \mathbb{T}$.

Proof. Let $I=\left[\alpha^{\prime}, \alpha^{\prime \prime}\right], \Delta=\left[E^{\prime}, E^{\prime \prime}\right]$ and $R=I \times \Delta$. Let $Y$ be the union of all rectangles $Q=\left[\alpha_{Q}^{\prime}, \alpha_{Q}^{\prime \prime}\right] \times\left[E_{Q}^{\prime}, E_{Q}^{\prime \prime}\right]=I_{Q} \times \Delta_{Q}$ with rational vertices, such that for all $\alpha \in I_{Q} \backslash N_{Q}$, where $\left|N_{Q}\right|=0(|\cdot|$ denotes the Lebesgue measure), the operator $A_{\omega}^{\alpha}$ has Cantor spectrum on $\Delta_{Q}$ and a positive Lyapunov exponent a.e. on $\Delta_{Q}$. It follows from the proof of Theorem 6 (and the fact that almost all $\alpha$ are Diophantine in the sense of (20)) that $|R \backslash Y|=0$. In addition, $\left|\bigcup_{Q} N_{Q}\right|=0$. This implies that for a.e. $\alpha \in I$ the interval $\Delta$ is almost entirely covered with countably many intervals on each of which the operator $A_{\omega}^{\alpha}$ has Cantor spectrum and a.e. positive Lyapunov exponent; therefore, the same is true for the whole interval $\Delta$. Consequently, for a.e. $\alpha \in I$ the spectrum of $A_{\omega}^{\alpha}$ on $\Delta$ is singular for all $\omega$ and, for generic $\omega$, singular continuous.

Remark. Another sufficient condition for the operator (19) to have Cantor spectrum was given by Sinai [23]. 
6.2. Rank one perturbations. Consider an operator family

$$
A_{t}=A+t P, \quad t \in \mathbb{R},
$$

where $A$ is a cyclic self-adjoint operator and $P$ is an orthogonal projection onto its one-dimensional cyclic subspace $\mathbb{C} \varphi(\|\varphi\|=1): P y=(y, \varphi) \varphi$. All operators $A_{t}$ have the same essential spectrum, which we denote by $\Sigma_{\text {ess. }}$. It was proved in [12] and [7] (in different ways) that for a generic $t$ there are no eigenvalues of $A_{t}$ in $\Sigma_{\mathrm{ess}}$.

The first and easiest step in both proofs (especially in [7]) was to show that the set

$$
\left\{E \in \Sigma \mid E \text { is an eigenvalue of } A_{t} \text { for some } t\right\}
$$

is meager. Now we will show how to replace the rest of the proof by a reference to Theorem 3.

We apply Theorem 3 with $S=\mathbb{R}$ and $F=\Sigma_{\text {ess. }}$. Assumptions (a) and (b) of Theorem 3 are fulfilled; to prove (c), assume first that $E$ and $\widetilde{E}$ are eigenvalues of the operators $A_{t}$ and $A_{\tilde{t}}$, respectively, with nonzero eigenvectors $y$ and $\tilde{y}$. Since the vector $\varphi$ is cyclic for $A$, it is easy to see that $(y, \varphi) \neq 0$ and $(\tilde{y}, \varphi) \neq 0$, so we may assume that $(y, \varphi)=(\tilde{y}, \varphi)=1$. Then the obvious identity

$$
(\tilde{E}-E)(\tilde{y}, y)=(\tilde{t}-t)(\tilde{y}, P y)
$$

takes the form

$$
(\widetilde{E}-E)(\tilde{y}, y)=\tilde{t}-t,
$$

so that $\tilde{t}=t$ if $\tilde{E}=E$. Therefore, for any $E \in \mathbb{R}$ the set

$$
N_{E}:=\left\{t \in \mathbb{R}: E \text { is an eigenvalue of } A_{t}\right\}
$$

contains at most one point, which proves (c).

6.3. Even almost periodic potentials. Let $v(t)$ be a real-valued almost periodic function on $\mathbb{R}, S$ its Bohr compact, and $A_{s}=-d^{2} / d t^{2}+v_{s}(t)(s \in S)$ the corresponding family of Schrödinger operators in $L^{2}(\mathbb{R})$. It was proved in [15] that if $v(t)$ is even, then for a generic $s \in S$ the operator $A_{s}$ has no eigenvalues. (The $l^{2}(\mathbb{Z})$ version of this result was also proved in [15].)

Now we will show how a somewhat more general statement can be derived from the results of the present work.

Let $S$ be a complete metric space; $\left\{G^{t}\right\}_{t \in \mathbb{R}}$ a group of its homeomorphisms such that $G^{t}(s)$ is continuous in $t$ for any $s$; and $V(\cdot)$ a bounded continuous real-valued function on $S$. For $s \in S$, define a self-adjoint operator $A_{s}$ on $L^{2}(\mathbb{R})$ by

$$
A_{s}=-\frac{d^{2}}{d t^{2}}+v_{s},
$$

where $v_{s}$ is the operator of multiplication by the function

$$
v_{s}(t)=V\left(G^{t}(s)\right) \text {. }
$$


Theorem 7. Suppose that for some $s_{0} \in S$, setting $\gamma(t):=G^{t}\left(s_{0}\right)$, we have:

(a) $V(\gamma(-t))=V(\gamma(t))$ for all $t \in \mathbb{R}$;

(b) the trajectory $\{\gamma(t)\}_{t \in \mathbb{R}}$ of $s_{0}$ is dense in $S$;

(c) there exists a sequence $t_{k}\left(\left|t_{k}\right| \rightarrow \infty\right)$ for which $\gamma\left(t_{k}\right) \rightarrow s_{0}$.

Then there is a dense $G_{\delta}$ set $S_{0} \subset S$ such that for all $s \in S_{0}$ the operator (24), (25) has no eigenvalues.

Proof. Apply Theorem 2 with $F=\mathbb{R}$. Assume that case (ii) of the theorem takes place. Pick a point $s^{*} \in Q$; denoting $y_{s^{*}}$ by $y^{*}$, we have: for any $\delta>0$ there exists a neighborhood $U^{\prime}$ of $s^{*}\left(U^{\prime} \subset U\right)$ such that $\left\|y_{s}-y^{*}\right\| \leq \delta$ for any $s \in U^{\prime}$.

In view of (b) and (c), we can choose a sequence $u_{k} \in \mathbb{R}$ so that $\left|u_{k}\right| \rightarrow \infty$ and $s_{k}:=\gamma\left(u_{k}\right) \in U^{\prime}$ for all $k$. Then we have

$$
\left\|y_{s_{k}}-y^{*}\right\| \leq \delta, \quad k=1,2, \ldots
$$

The function $y_{s_{k}}(t)$ is a solution of the equation $-y^{\prime \prime}+v_{s_{k}}(t) y=\varepsilon_{s_{k}} y$, or equivalently, since $v_{s_{k}}(t)=V\left(G^{t}\left(s_{k}\right)\right)=V\left(\gamma\left(t+u_{k}\right)\right)$, the equation

$$
-y^{\prime \prime}+v\left(t+u_{k}\right) y=\varepsilon_{s_{k}} y,
$$

where $v(t)=V(\gamma(t))$. It follows that the function $y_{s_{k}}(-t)$ solves the "reflected" equation $-y^{\prime \prime}+v\left(-t+u_{k}\right) y=\varepsilon_{s_{k}} y$, or

$$
-y^{\prime \prime}+v\left(t-u_{k}\right) y=\varepsilon_{s_{k}} y
$$

(here we use the evenness of the function $v(\cdot)$, which follows from (a)).

Since the equation (28) is the $\left(-2 u_{k}\right)$-shift of (27) and the $L^{2}$-solution of each of these equations is uniquely determined up to a constant factor, we have

$$
y_{s_{k}}(-t)=c_{k} y_{s_{k}}\left(t-2 u_{k}\right) \text {, }
$$

where $\left|c_{k}\right|=1$.

It follows from (29) and (26) that

$$
\left\|y^{*}(-t)-c_{k} y^{*}\left(t-2 u_{k}\right)\right\| \leq 2 \delta, \quad k=1,2, \ldots
$$

But for large $k,\left|2 u_{k}\right|$ is large, so the functions $y^{*}\left(t-2 u_{k}\right)$ and $y^{*}(-t)$ are almost orthogonal, and hence passing to the limit we have $2\left\|y^{*}\right\|^{2} \leq 4 \delta^{2}$. Since $\delta>0$ can be chosen arbitrarily small, we obtain: $y^{*}=0$. We have arrived at a contradiction, which shows that case (ii) of Theorem 2 is impossible.

Corollary 9. Let $v: \mathbb{R} \rightarrow \mathbb{R}$ be a bounded uniformly continuous function, and $S$ be the set of all pointwise limits of its shifts $v_{u}(t)=v(t+u)(u \in \mathbb{R})$ endowed with the topology of pointwise convergence (so that $S$ is a compact metrizable topological space). Suppose that $v(-t)=v(t)$ for all $t \in \mathbb{R}$ and $v\left(t+u_{n}\right) \rightarrow v(t)$ for some sequence $u_{n} \in \mathbb{R}\left(\left|u_{n}\right| \rightarrow \infty\right)$ and all $t \in \mathbb{R}$. Then for a generic $s \in S$ the operator $-d^{2} / d t^{2}+s(t)$ in $L^{2}(\mathbb{R})$ does not have eigenvalues. 
Proof. Define homeomorphisms $G^{t}(t \in \mathbb{R})$ of $S$ by $\left(G^{t}(s)\right)(u)=s(u+t)$ and the function $V$ on $S$ by $V(s)=s(0)$. Then the function (25) is $v_{s}(t)=V\left(G^{t}(s)\right)=$ $\left(G^{t}(s)\right)(0)=s(t)$, and it remains to apply Theorem 7 with $s_{0}=v(\cdot)$.

In the particular case, where $v(t)$ is an even almost periodic function, we obtain the result [15].

The $l^{2}(\mathbb{Z})$ versions of these results can be proved similarly.

\section{References}

[1] A. Avila, J. Bochi, and D. Damanik, Cantor spectrum for Schrödinger operators with potentials arising from generalized skew-shifts. Duke Math. J. 146 (2009), 253-280. MR 2477761 Zbl 1165.37012

[2] A. Avila and S. Jitomirskaya, The ten Martini problem. Ann. of Math. (2) 170 (2009), 303-342. MR 2521117 Zbl 1166.47031

[3] J. Bourgain, Green's function estimates for lattice Schrödinger operators and applications. Annals of Mathematics Studies 158. Princeton University Press, Princeton, NJ, 2005. MR 2100420 Zbl 1137.35001

[4] J. Bourgain and S. Jitomirskaya, Continuity of the Lyapunov exponent for quasiperiodic operators with analytic potential. Dedicated to David Ruelle and Yasha Sinai on the occasion of their $65^{\text {th }}$ birthdays. J. Stat. Phys. 108 (2002), 1203-1218. MR 1933451 Zbl 1039.81019

[5] R. Carmona and J. Lacroix, Spectral theory of random Schrödinger operators. Birkhäuser, Boston, MA, 1990. MR 1102675 Zbl 0717.60074

[6] H. L. Cycon, R. G. Froese, W. Kirsch, and B. Simon, Schrödinger operators with application to quantum mechanics and global geometry. Springer, Berlin etc., 1987. MR 0883643 Zbl 0619.47005

[7] R. del Rio, N. Makarov, and B. Simon, Operators with singular continuous spectrum II. Rank one operators. Comm. Math. Phys. 165 (1994), 59-67. MR 1298942 Zbl 1055.47500

[8] I. M. Glazman, Direct methods of qualitative spectral analysis of singular differential operators. Israel Program for Scientific Translations, Jerusalem, 1965. Daniel Davey \& Co., New York, 1966. MR 0190800

[9] I. Ja. Goldsheid, Asymptotics of the product of random matrices depending on a parameter. Dokl. Akad. Nauk SSSR 224 (1975), 1248-1253 (in Russian).

[10] I. Ja. Goldsheid, Asymptotic properties of the product of random matrices depending on a parameter. In R. L. Dobrushin and Ya. G. Sinai (eds.), Multicomponent random systems. Nauka, Moscow, 1978 (in Russian). English transl. Advances in Probability and Related Topics 6. Marcel Dekker, New York, 1980, 239-283. MR 0599538 Zbl 0443.60100

[11] M. Goldstein, and W. Schlag, On resonances and the formation of gaps in the spectrum of quasi-periodic Schrödinger equations. Ann. of Math. (2) 173 (2011), 337-475. MR 2753606 Zbl 05960662 
[12] A. Ya. Gordon, Pure point spectrum under 1-parameter perturbations and instability of Anderson localization. Comm. Math. Phys. 164 (1994), 489-505. MR 1291242 Zbl 0839.47002

[13] A. Ya. Gordon, Purely continuous spectrum for generic almost-periodic potential. In E. Carlen, E. M. Harrell and M. Loss (eds.), Advances in differential equations and mathematical physics. Papers from the International Conference on Differential Equations and Mathematical Physics held at the Georgia Institute of Technology, Atlanta, GA, March 23-29, 1997. Contemporary Mathematics 217. Amer. Math. Soc., Providence, RI, 1998, 183-189. MR 1606717 Zbl 0901.34079

[14] S. Ya. Jitomirskaya, Metal-insulator transition for the almost Mathieu operator. Ann. of Math. (2) 150 (1999), 1159-1175. MR 1740982 Zbl 0946.47018

[15] S. Ya. Jitomirskaya and B. Simon, Operators with singular continuous spectrum III. Almost periodic Schrödinger operators. Comm. Math. Phys. 165 (1994), 201-205. MR 1298948 Zbl 0830.34074

[16] Y. Last, Spectral theory of Sturm-Liouville operators on infinite intervals: A review of recent developments. In W. O. Amrein, A. M. Hinz, and D. B. Pearson (eds.), SturmLiouville theory. Past and present. Including papers from the International Colloquium held at the University of Geneva, Geneva, September 15-19, 2003. In Sturm-Liouville theory. Birkhäuser Verlag, Basel, 2005, 99-120. MR 2145079 Zbl 1098.39011

[17] Y. Last and B. Simon, Eigenfunctions, transfer matrices, and absolutely continuous spectrum of one-dimensional Schrödinger operators. Invent. Math. 135 (1999), 329-367. MR 1666767 Zbl 0931.34066

[18] J. Oxtoby, Measure and category. Second edition. Graduate Texts in Mathematics 2. Springer Verlag, New York etc., 1980. MR 0584443 Zbl 0435.28011

[19] L. Pastur and A. Figotin, Spectra of random and almost-periodic operators. Grundlehren der Mathematischen Wissenschaften 297. Springer Verlag, New York, 1992. MR 1223779 Zbl 0752.47002

[20] M. Reed and B. Simon, Methods of modern mathematical physics I. Functional analysis. Academic Press, New York and London, 1972. MR 0493419 Zbl 0459.46001

[21] B. Simon, Operators with singular continuous spectrum I. General operators. Ann. of Math. (2) 141 (1995), 131-145. MR 1314033 Zbl 0851.47003

[22] B. Simon and T. Wolff, Singular continuous spectrum under rank one perturbations and localization for random Hamiltonians. Comm. Pure Appl. Math. 39 (1986), 75-90. MR 0820340 Zbl 0609.47001

[23] Ya. G. Sinai, Anderson localization for one-dimensional difference Schrödinger operator with quasi-periodic potential. J. Stat. Phys. 46 (1987), 861-909. MR 0893122

Received April 29, 2011; revised June 20, 2012

Alexander Y. Gordon, Department of Mathematics and Statistics, University of North Carolina at Charlotte, 9201 University City Blvd, Charlotte, NC 28223, U.S.A.

E-mail: aygordon@uncc.edu 